Glycoconjugate Journal 19, 363-368, 2003

\title{
Developmental roles of heparan sulfate proteoglycans in Drosophila*
}

\author{
Xinhua Lin ${ }^{1}$ and Norbert Perrimon ${ }^{2}$ \\ ${ }^{1}$ Division of Developmental Biology, Children's Hospital Medical Center, Cincinnati, OH 45229, USA, ${ }^{2}$ Department of Genetics, \\ Howard Hughes Medical School, Harvard Medical School, Boston, MA 02115, USA
}

\begin{abstract}
The formation of complex patterns in multi-cellular organisms is regulated by a number of signaling pathways. In particular, the Wnt and Hedgehog $(\mathrm{Hh})$ pathways have been identified as critical organizers of pattern in many tissues. Although extensive biochemical and genetic studies have elucidated the central components of the signal transduction pathways regulated by these secreted molecules, we still do not understand fully how they organize gradients of gene activities through field of cells. Studies in Drosophila have implicated a role for heparan sulfate proteoglycans (HSPGs) in regulating the signaling activities and distribution of both $\mathrm{Wnt}$ and $\mathrm{Hh}$. Here we review these findings and discuss various models by which HSPGs regulate the distributions of Wnt and Hh morphogens.
\end{abstract}

Published in 2003.

Keywords: heparan sulfate proteoglycan, Wnt, Wingless, Hedgehog, Drosophila

\section{Introduction}

During development of multi-cellular organisms, cell fate determination is controlled by the integration of several signaling systems that are initiated by several evolutionarily conserved extracellular molecules. These include members of Wnt, Hedgehog (Hh), transforming growth factor- $\beta$ (TGF- $\beta$ ) and fibroblast growth factor (FGF) families. Among them, members of the Wnt, Hh and TGF- $\beta$ families have been shown to function as morphogens; i.e., they are expressed in restricted regions of tissues and can form long-range concentration gradients to specify different cell fates [1,2]. During the past several decades, both biochemical and genetic studies have elucidated the central components of the signal transduction pathways regulated by these molecules. However, less is known about their distribution through tissues and how they act long range to organize pattern.

In the last few years, our laboratory as well as others have conducted in vivo functional studies of HSPGs in Drosophila. These studies have demonstrated that they play critical roles in regulating the signaling activities of Wnt, Hh, TGF- $\beta$ and

To whom correspondence should be addressed: Xinhua Lin, PhD, Division of Developmental Biology, Cincinnati Children's Hospital Medical Center, 3333 Burnet avenue, Cincinnati, OH 45229, USA. Tel.: (513)636-2144; Fax: (513)-636-4317; E-mail: linyby@chmcc.org

*This review is dedicated to the memory of Merton Bernfield, our colleague and friend who taught us so much about proteoglycans.
FGF molecules. Further, it has become clear that the distribution of Wnt and Hh morphogens is regulated by HSPGs. This review will focus on the molecular mechanism(s) by which HSPGs regulate the signaling activity and the extracellular distribution of Wnt and Hh molecules. For other aspects of the functions of HSPGs in development, see previous reviews [3-8].

2. HSPGs: A family of evolutionarily conserved molecules HSPGs are extracellular matrix and cell surface macromolecules that consist of a protein core to which HS glycosaminoglycan (GAG) chains are attached (see Introduction by Silbert and Sugumaran in this issue). These molecules can be classified into several families based on the structure of the protein cores. Glypicans and syndecans are two major cell surface HSPGs, both of which are integral membrane proteins. Glypicans are disulfide-stabilized globular proteins linked to the plasma membrane by a glycosylphosphatidylinositol linkage. Syndecans are transmembrane proteins that contain a highly conserved short [34-38 residue] carboxy-terminal cytoplasmic domain. While glypicans bear exclusively HS chains, syndecans may be decorated with both HS and chondroitin sulfate (CS). Besides syndecan and glypicans, perlecan is a secreted HSPG that is mainly distributed in the extracellular matrix, and has exclusively HS chains [6]. 
Biosynthesis of HS is initiated in the Golgi at its attachment site to a core protein, which contains between 2 and 4 Ser-Gly sequences. An oligosaccharide primer is first attached to specific serine residues on a proteoglycan core protein. This primer serves as a substrate for the co-polymerases that add alternating linked D-glucuronic acid (GlcA) and N-acetylD-glucosamine (GlcNAc) residues, to form chains of about 100 or more sugar units in length. This polymer is subsequently modified through a series of reactions, requiring both $\mathrm{N}$-deacetylation and $\mathrm{N}$-sulfation of GlcNAc units, epimerization of GlcA to L-iduronic acid (IdoA) residues, and O-sulfation at various positions. From these modifications, enormous structural heterogeneity in HS structure can be produced (see introduction by Silbert and Sugumaran in this issue).

Both core proteins and enzymes involved in the biosynthesis of HS are conserved in vertebrates and invertebrates [6,8]. In Drosophila, a single syndecan gene, two glypican genes, and a perlecan homolog have been identified [6,8]. So far, functional studies are only available for the two glypican family members, division abnormally delayed (dally) [9], and dally-like (dly) $[10,11]$ (see below). Drosophila homologs of most of the vertebrate enzymes involved in HS biosynthesis are also present in the fly genome. These include specific sugar transporters, enzymes involved in the initiation of oligosaccharide primer and specific modifications $[3,6]$. In the past few years, some of the enzymes that participate in HS biosynthesis have been identified and genetically characterized in Drosophila [4,6] see below). Others such as xylosyltransferase [12] and a EXT 3-like glycosyltransferase [13], have been biochemically characterized recently.

\section{Identification and isolation of mutations in HS enzymes and core proteins}

Mutations in the genes encoding some of the enzymes involved in the biosynthesis of HS were initially identified from our genetic screen designed to characterize the maternal effects of zygotic lethal mutations [14,15]. From this genetic screen, we have isolated several new segment polarity genes that exhibit mutant phenotypes that resemble those associated with loss of either wingless $(w g)$ or $h h$ activity. These genes are sugarless $(s g l)[4,16]$, sulfateless $(s f)[4,17,18]$ and tout velu $(t t v)[19,20]$, which encode Drosophila homologs of UDP-D-glucose dehydrogenase, HS N-deacetylase/N-sulfotransferase and HS polymerase EXT-1, respectively. dally was isolated from a different genetic screen targeted to obtain mutants affecting cell division patterning in the developing central nervous system of Drosophila [9,21]. The identification of mutations in these genes has provided the starting materials for analyzing the function of these HSPGs. Below, we review our analysis of HSPGs in the context of Wnt and Hh signaling. Further, we discuss the various mechanisms by which HSPGs may regulate the signaling activities and protein distributions of the $\mathrm{Wg}$ and $\mathrm{Hh}$ morphogens.

\section{Role of HSPGs in Wg signaling and morphogen gradient formation}

\subsection{Wg: A morphogen critical in development}

Wnt proteins are members of a large family of secreted, cysteine-rich glycoproteins that act as ligands, and stimulate receptor-mediated signal transduction pathways in both vertebrates and invertebrates [22]. All Wnts contain a signal sequence, 22 conserved cysteine residues, and multiple potential $\mathrm{N}$-linked glycosylation sites. Wnt proteins are poorly secreted into the culture medium and are tightly associated with the cell surface and the extracellular matrix. Thus, how Wnt proteins travel from cell to cell to initiate intercellular signaling is of particular interest. The homolog of vertebrate Wnt-1 is encoded by Drosophila $\mathrm{Wg}$, which has been implicated as a mediator of positional information in a wide variety of developmental contexts. These include segmentation of the epidermis, patterning of the midgut epithelium, formation of the stomatogastric nervous system, neuroblast determination and differentiation and patterning of imaginal discs $[22,23]$. Wg can exert both shortand long-range effects during the development of the embryo and imaginal discs. The function of $\mathrm{Wg}$ protein has been particularly well explored in embryonic patterning of epidermis and wing patterning during larval development.

$\mathrm{Wg}$ acts both as a short-range inducer and a long-range morphogen during differentiation of the embryonic epidermis (Figure 1). Early in embryogenesis at stage 9-10, $w g$ is

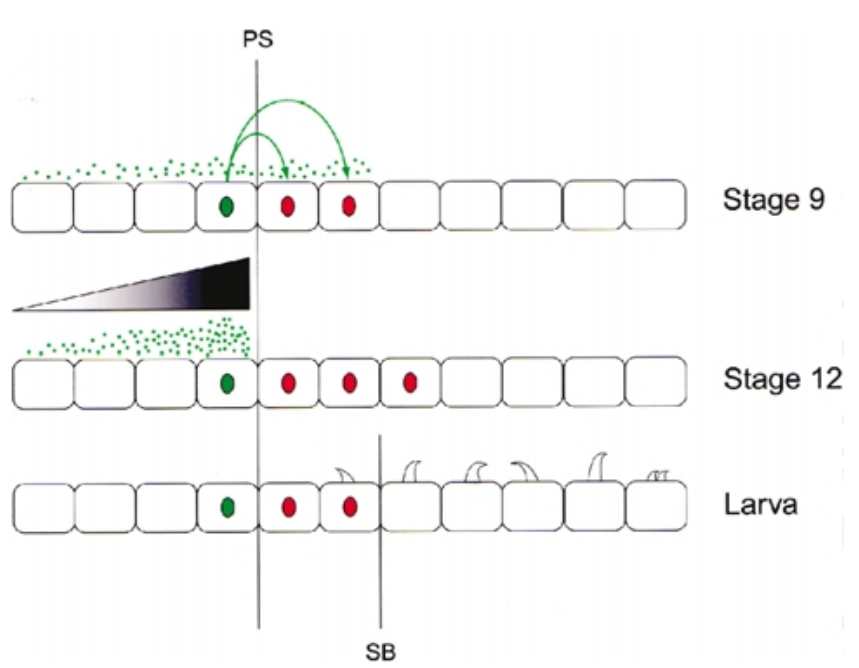

Figure 1. Roles of Wg signaling during embryonic segmentation in the ventral epidermis. During embryogenesis, $w g$ and en are expressed in stripes within each segment. The single row of wg-expressing cells is shown in green and en-expressing cells are in red. en is expressed in cells posterior to the $\mathrm{Wg}$ stripe. At stage $9, \mathrm{Wg}$ protein is distributed symmetrically and acts shortrange posteriorly to maintain en expression. At stage $12, \mathrm{Wg}$ protein is mainly distributed anteriorly and forms a morphogen gradient that is required for producing the naked cuticle. The position of the segmental border (SB) and the parasegmental boundary (PS) are indicated. 
expressed in stripes of epidermal cells that are immediately adjacent, and anterior, to cells expressing the homeobox gene engrailed (en). Wg functions at short-range to maintain the expression of en. Subsequently, En, through a signaling pathway mediated by $\mathrm{Hh}$ is required for the maintenance of $w g$ transcription [24]. The juxtaposition of $e n / h h$ and $w g$-expressing cells is crucial for the formation of alternating bands of naked cuticle and denticles within each segmental unit (Figure 1). At a later stage (stage 12), $\mathrm{Wg}$ distributes anteriorly to its expressing cells and forms a concentration gradient required for producing the "naked cuticle" that lacks denticle bands $[24,25]$. Thus, Wg functions as a long-range morphogen that gives positional information to the anterior cells in front of the source.

The function of $\mathrm{Wg}$ as a morphogen is best studied in the wing imaginal disc where it is expressed in a narrow strip of cells at the dorso-ventral compartment border (Figure 2). $\mathrm{Wg}$ acts as short-range inducer to specify cell fates at the dorso-ventral boundary. Wg also acts up to 20-30 cell diameters away from its site of synthesis to activate the transcription of downstream target genes in a dose-dependent manner, which is a characteristic of morphogen molecules. The extracellular Wg protein gradient can be visualized on the basolateral surface of the wing imaginal disc epithelium [26]. $\mathrm{Wg}$ protein moves rapidly to form a long-range extracellular gradient. It has been proposed that the $\mathrm{Wg}$ protein gradient is established by rapid diffusion through the extracellular space, depending on continuous secretion and rapid turnover $[1,26]$.
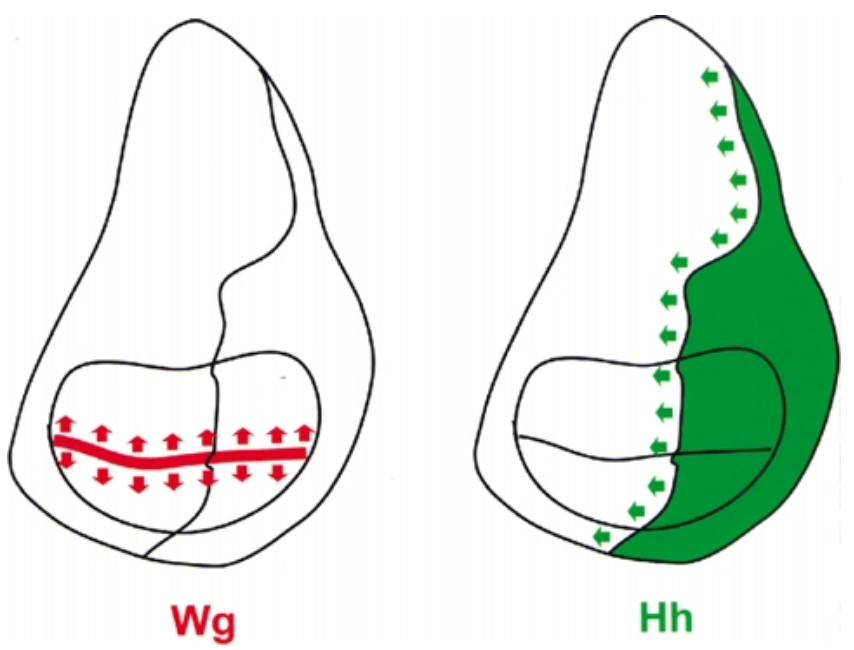

Figure 2. Roles of $\mathrm{Wg}$ and $\mathrm{Hh}$ in patterning of the wing imaginal disc. In a third instar larvae wing imaginal disc, Wg (red) is expressed at the dorso-ventral border of the wing disc (A) and signals dorsally (arrows up) and ventrally (arrows down) to organize patterning. Hh (green) is expressed in the posterior compartment and moves in the anterior compartment of the disc to control anteroposterior axis formation.
4.2. HS is required for $\mathrm{Wg}$ signaling and its extracellular distribution

Our genetic analyses have provided evidence for an in vivo function of $\mathrm{HS}$ in Wg signaling and distribution. Both $s g l$ and $s f$ were initially identified and isolated as genes involved in $\mathrm{Wg}$ signaling since their mutants exhibit defects of cuticle patterning similar to that of $w g$ mutant $[16,17]$. In $s g l$ and $s f l$ mutant embryos, we also observed other defects associated with $\mathrm{Wg}$ dependent developmental processes including the development of the somatogastric nervous system and midgut constriction $[16,17]$. Further, we have obtained genetic evidence for the requirement of $\mathrm{HS}$ in both $\mathrm{Wg}$ short- and long-range signaling in wing imaginal disc [17]. In the wing discs homozygous for $s f$ mutant, the expression of the short-range target-gene neuralized is abolished, and the expression of the long-range target-gene distaless $(d l l)$ is markedly reduced. Furthermore, analysis of $s f$ provided direct evidence for a role of HS in the distribution of extracellular Wg [11] since in $s f$ mutant clones, the distribution of extracellular $\mathrm{Wg}$ is dramatically reduced. Altogether, these results point to a role for $\mathrm{HS}$ in most, if not all, of the $\mathrm{Wg}$ dependent developmental processes. Mechanistically, at least one of the mechanisms of the HS action in $\mathrm{Wg}$ signaling is to regulate the extracellular distribution of $\mathrm{Wg}$ [11].

\subsection{Drosophila glypicans, Dally and Dly, are involved in $\mathrm{Wg}$ signaling}

We have demonstrated that the two Drosophila glypicans, dally and dly, are involved in Wg signaling. First, hypomorphic dally alleles exhibit some wing margin defects [9], a phenotype reminiscent of partial loss of $w g$ activity. The wing defects associated with dally mutants can be enhanced by a reduction in $\mathrm{Wg}$ protein or a reduction in $\mathrm{Wg}$ signaling activity, but can be suppressed by introducing an activated downstream Wg signaling component [17]. Second, we also observed that dally mutants can suppress gain-of-function phenotype induced by ectopic expression of the Wg receptor Drosophila frizzled 2 (Dfz2). These results suggest that Dally is a major HSPG involved in $\mathrm{Wg}$ signaling in wing imaginal disc.

We have also demonstrated that Dally and Dly are required for cuticle patterning in embryogenesis. Disruptions of either dally or dly by RNA interference (RNAi) in embryos, produced patterning defects in the cuticle that are similar to the defects associated with weak $w g$ mutants. Interestingly, more severe cuticle patterning defects were observed in embryos injected with dly dsRNA than those injected with dally RNAi, suggesting that Dly may play a more important role than Dally in Wg signaling - at least in the ventral epidermis. Furthermore, we also observed that many embryos injected with dly dsRNA develop cuticles that lack naked cuticle but which are similar in size to wild-type embryos [11]. Since Wg signaling during late embryogenesis is required for producing naked cuticle (Figure 1), these results suggest that Dly is likely to be involved in Wg signaling at later developmental stages. Consistent with 
this, we have observed that $d l y$ transcripts are enriched in cells anterior to the $w g$-expressing cells [11].

Consistent with our results on the role of Dally and Dly in Wg signaling, recently, in both S. Cohen and K. Basler labs, they identified a gene called notum or wingful, which encodes a protein to function as a repressor for $\mathrm{Wg}$ signaling [27]. It encodes a member of the alpha/beta-hydrolase superfamily, with similarity to pectin acetylesterases, and influences $\mathrm{Wg}$ protein distribution by modifying the HSPGs Dally-like and Dally [27], further suggesting important roles of Dally and Dly in $\mathrm{Wg}$ signaling.

\subsection{Molecular mechanism(s) by which HSPGs may regulate} $\mathrm{Wg}$ signaling and its distribution

Our genetic analyses in both HS enzymes ( $s g l$ and $s f$ ) and glypicans (dally and dly) have provided strong evidence for a role of HSPG in Wg signaling. Our results also suggest that dally and dly are the major HSPGs involved in $\mathrm{Wg}$ signaling. In the following section, we propose several mechanisms by which HSPGs may regulate Wg signaling and its distribution.

\section{Concentration model}

In this model, the function of HSPG is to increase the local concentration of Wg ligand for its receptor. HSPGs bind to Wg and reduce the dimensionality of $\mathrm{Wg}$ ligand diffusion from three to two dimensions. In the absence of HSPGs, the concentration of $\mathrm{Wg}$ protein may be lower than its threshold concentration and the efficiency of $\mathrm{Wg}$ signaling will be reduced. Several genetic experiments support this model. First, we have observed that $\mathrm{Wg}$ signaling is not completely abolished in the formation of somatogastric nervous system and Malpighian tubules [16]. Perhaps, the threshold concentration of Wg signaling required for these tissues is relatively low. Second, in the ventral epidermis, over-expression of ectopic $\mathrm{Wg}$ protein can compensate for the loss of Wg signaling to partially rescue the segmentation defects associated with $s f$ and $s g l[16]$.

\section{Trapping/retention model}

HSPGs are required for trapping/retention of $\mathrm{Wg}$ protein in receiving cells. This model is based on the following observations. (1) HSPGs are required for the distribution of extracellular $\mathrm{Wg}$ protein in imaginal disc [11]. (2) Ectopic expression of Dly in wing imaginal disc leads to an accumulation of $\mathrm{Wg}$ protein [11]. (3) Embryos devoid of Dly by RNAi develop segmentation defects that lack naked cuticle, but with relatively normal sized embryos, suggesting that Dly is involved in long-range $\mathrm{Wg}$ patterning required for generating naked cuticle [11]. According to this model, $\mathrm{Wg}$ moves through rapid diffusion to receiving cells where $\mathrm{Wg}$ is trapped by the abundant HSPGs. HSPGs stabilize Wg by either limiting its diffusion or by preventing it from being degraded by extracellular proteases. We propose that both Dally and Dly are involved in $\mathrm{Wg}$ morphogen gradient formation in wing disc since both are expressed in this tissue. It is also worth noting that both Drosophila syndecan and perlecan are expressed in wing disc (Lin et al. unpublished result) raising the possibility that these molecules contribute to formation of the Wg morphogen gradient as well.

As mentioned earlier, in the epidermis of stage 12 embryo, $\mathrm{Wg}$ functions as a morphogen that distributes anteriorly and forms a concentration gradient required for producing "naked cuticle" (Figure 1). Two different mechanisms were proposed by which $\mathrm{Wg}$ acts $[25,28]$. In the first model, it was proposed that the $\mathrm{Wg}$ gradient is established by cell spreading [28]. The cells leaving the expression domain retain inherited $\mathrm{Wg}$ protein in secretory vesicles and carry it forwards over a distance of up to four cell diameters. Experiments using a membranetethered form of $\mathrm{Wg}$ showed that this mechanism is sufficient to account for the normal range of $\mathrm{Wg}$. In the second model, $\mathrm{Wg}$ can also reach distant target cells independently of protein inheritance, possibly by restricted diffusion. It is important to note that in either case retention of $\mathrm{Wg}$ in anterior cells is critical for producing naked cuticle cell fate. In this regard, Dly may be involved in the retention of $\mathrm{Wg}$ protein in these cells. If that is the case, a reduction of Dly may reduce the ability of anterior cells to retain $\mathrm{Wg}$ and thus fail to produce naked cuticle. Consistent with this, embryos injected with $d l y$ dsRNA develop cuticle that lacks denticle bands. Further detailed analysis of $d l y$ mutant embryos will be required to validate this model.

\section{Transport model}

Recently, Eaton and her colleagues reported an exciting finding that supports a novel cell biological mechanism for transporting Wg through a field of cells [29]. When a glycosylphosphatidyl inositol-anchored green fluorescent fusion protein was expressed in different subsets of the imaginal disc using the Gal4/UAS system, fluorescence was observed not only on the plasma membrane of expressing cells, but also in punctate structures in non-expressing cells. They have called these punctate particles "argosomes" because of their ability to travel. Several lines of evidence suggest that $\mathrm{Wg}$ can travel over the disc epithelium on argosomes. First, Wg-expressing cells produce argosomes from basolateral membranes that contain high levels of $\mathrm{Wg}$ proteins. Second, in receiving cells, $\mathrm{Wg}$ co-localizes with argosomes that are derived from Wg-expressing cells. Finally the rate at which argosomes spread through the disc epithelium is similar to the rate at which $\mathrm{Wg}$ travels.

Interestingly, following treatment with either heparinase I or III, Wg was almost undetectable on the plasma membrane of both $\mathrm{Wg}$-expressing cells and the surrounding tissue. This result is consistent with our observation that extracellular $\mathrm{Wg}$ protein is strikingly reduced in clones of $s f$ mutant cells $[11,29]$. Although the production of argosomes is apparently independent of the presence of HSPGs and Wg protein, both Wg and HSPGs could be present in argosomes. It is important to note that argosomes were observed by green fluorescent protein anchored with glycosylphosphatidyl inositol linker, and that both Dally 
and Dly are glycosylphosphatidyl inositol-anchored proteoglycans suggesting that these HSPGs are present in argosomes.

\section{HSPGs are involved in Hh movement}

Like Wnts, members of Hh family of secreted signaling molecules have also been shown to act in the specification of cell fate and patterning in many developmental processes [30]. Hh is made as a precursor protein that is autocatalytically cleaved to produce an $\mathrm{N}$-terminal (Hh-N) and a C-terminal (HhC) fragment [30]. During this cleavage, a cholesterol moiety is covalently attached to the last amino acid of Hh-N to create biologically active processed $\mathrm{Hh}-\mathrm{N}$ (Hh-Np) [30]. HhNp lacks the properties of a diffusible molecule when it is associated to a cholesterol moeity.

Our understanding of the role of HSPGs in Hh signaling mainly comes from the analysis of the ttv mutant phenotype [19]. As mentioned before, ttv encodes a Drosophila homolog of the human EXT-1 tumor suppressor protein that has been biochemically characterized as a HS polymerase involved in HS biosynthesis $[19,20]$. Analysis of $t t v$ mutant clones in the wing disc led to the conclusion that HSPGs are involved in Hh movement. As shown in Figure 2, in the Drosophila wing disc, $h h$ is expressed in the posterior compartment. Hh travels to the anterior compartment and acts at a distance of 8-10 cell diameters from the site of its production to induce the expression of its target gene patched (ptc) and decapentaplegic $(d p p)$, and stabilizes the Cubitus Interruptus $(\mathrm{Ci})$ protein along the anteroposterior boundary [30]. Homozygous mutant clones of $t t v$ along this boundary show reduced ptc expression and $\mathrm{Ci}$ stabilization [19]. Importantly, Hh diffusion occurs through a ptc mutant clone, since ectopic induction of ptc expression is found in wild-type cells localized in the anterior compartment distally to a clone of ptc mutant cells. While Hh protein can diffuse through the ptc mutant clone, this does not occur through a clone of ptc-ttv mutant cells, arguing that Ttv is required for Hh movement [19].

Recently, a novel patched-like transmembrane protein, Dispatched (Disp), has been identified and shown to act exclusively in Hh-secreting cells to liberate $\mathrm{HhNp}$ from either the internal or surface membrane of the cells [31]. A current model is that Disp is required in the posterior cells to transfer cholesterol-bound Hh to the Ttv-modified cell surface HSPGs in the anterior compartment $[31,32]$. The Hh-HSPG complex could either transfer Hh to its receptor or could form a Hh-HSPG-Ptc ternary complex in which HSPGs may function to stabilize a Hh-Ptc complex. In this regard, the HSPGs could also be directly involved in Hh signaling. In fact, in $t t v$ mutant embryos, $w g$ expression decays because of defective Hh signaling. Thus, even when Hh signals to immediate neighboring cells, the HSPGs may be required for regulation of $\mathrm{Ptc}$ by $\mathrm{Hh}$.

It is important to note that analysis of the $t t v$ mutant phenotype failed to reveal a function for Ttv in either the $\mathrm{Wg}$ or FGFs signaling pathways suggesting that Ttv is specific to $\mathrm{Hh}$ signaling [20]. This is unexpected as Ttv encodes a polymerase involved in HS chain biosynthesis, such that one would predict that it should have a phenotype similar to those of either $s g l$ or $s f$ mutants. Both qualitative and quantitative models have been proposed to explain this specificity. In the quantitative model, the biosynthesis of HSPG is reduced in the absence of Ttv activity but not completely eliminated. A reduced amount of HSPGs synthesized by other EXT enzymes may be sufficient for $\mathrm{Wg}$ and FGF signaling, but not for Hh signaling. Alternatively, in a qualitative model, specific EXTs may only modify a subset of protein cores or subset of HS to a specific protein core. HSPGs involved in Wg and FGF, but not in Hh signaling, may be modified properly in $t t v$ mutant embryos. In Drosophila, two additional EXT family members have been identified: brother of tout velu (botv) and sister of tout velu (sotv) that encode Drosophila EXT-3-like and EXT-2, respectively (Han and Lin, unpublished results). Further analysis of the phenotype associated with these genes will clarify the function of EXT proteins in $\mathrm{Hh}$ and $\mathrm{Wg}$ signaling.

\section{Conclusion}

Genetic studies in Drosophila have revealed the critical roles of HSPGs in regulating the activity and extracellular distribution of $\mathrm{Wg}$ and $\mathrm{Hh}$. Further detailed analysis of individual core proteins as well as enzymes involved in the modification of HS will be needed to define the precise mechanisms by which HSPG interact with extracellular factors. The combination of genetic, cell biological and biochemical approaches represents a powerful approach to characterize the function of HSPGs.

\section{Acknowledgments}

Work on HSPG in the laboratory of Norbert Perrimon (Investigator, Howard Hughes Medical Institute) is supported by the NIH and Mizutani foundation. Work on HSPG in Xinhua Lin's laboratory is supported partially by a NIH grant GM6389101A1, a grant from Ohio Cancer Associates and a start-up fund from Cincinnati Children Hospital Research Foundation.

\section{References}

1 Teleman AA, Strigini M, Cohen SM, Shaping morphogen gradients, Cell 105, 559-62 (2001).

2 Lawrence PA, Struhl G, Morphogens, compartments and pattern: Lessons from Drosophila? Cell 85, 951-61 (1996).

3 Baeg GH, Perrimon N, Functional binding of secreted molecules to heparan sulfate proteoglycans in Drosophila, Curr Opin Cell Biol 12, 575-80 (2000).

4 Lin X, Perrimon N, Role of heparan sulfate proteoglycans in cellcell signaling in Drosophila, Matrix Biol 19, 303-7 (2000).

5 Selva EM, Perrimon N, Role of heparan sulfate proteoglycans in cell signaling and cancer, Adv Cancer Res 83, 67-80 (2001).

6 Perrimon N, Bernfield M, Specificities of heparan sulfate proteoglycans in developmental processes, Nature 404, 725-8 (2000). 
7 Selleck SB, Genetic dissection of proteoglycan functions in Drosophila and C. elegans, Semin Cell Dev Biol 12, 127-34 (2001).

8 Lander AD, Selleck SB, The elusive functions of proteoglycans: In vivo veritas, J Cell Biol 148, 227-32 (2000).

9 Nakato H, Futch TA, Selleck SB, The division abnormally delayed (dally) gene: A putative integral membrane proteoglycan required for cell division patterning during post embryonic development of the nervous system in Drosophila, Development 121, 3687-702 (1995).

10 Khare N, Baumgartner S, Dally-like protein, a new Drosophila glypican with expression overlapping with wingless, Mech Dev 99, 199-202 (2000).

11 Baeg GH, Lin X, Khare N, Baumgartner S, Perrimon N, Heparan sulfate proteoglycans are critical for the organization of the extracellular distribution of Wingless, Development 128, 87-94 (2001).

12 Wilson IB, Functional characterization of Drosophila melanogaster peptide O-xylosyl transferase, the key enzyme for proteoglycan chain initiation and member of the core $2 / 1$ N-acetylglucosaminyl transferase family, J Biol Chem 277, 21207-12 (2002).

13 Kim BT, Kitagawa H, Tamura Ji J, Kusche-Gullberg M, Lindahl U, Sugahara K, Demonstration of a novel gene DEXT3 of Drosophila melanogaster as the essential $\mathrm{N}$-acetylglucosamine transferase in the heparan sulfate biosynthesis: Chain initiation and elongation, J Biol Chem 277, 13659-65 (2002).

14 Chou TB, Perrimon N, The autosomal FLP-DFS technique for generating germline mosaics in Drosophila melanogaster, Genetics 144, 1673-9 (1996).

15 Perrimon N, Lanjuin A, Arnold C, Noll E, Zygotic lethal mutations with maternal effect phenotypes in Drosophila melanogaster. II. Loci on the second and third chromosomes identified by Pelement-induced mutations, Genetics 144, 1681-92 (1996).

16 Hacker U, Lin X, Perrimon N, The Drosophila sugarless gene modulates Wingless signalling and encodes an enzyme involved in polysaccharide biosynthesis, Development 124, 3565-73 (1997).

17 Lin X, Perrimon N, Dally cooperates with Drosophila Frizzled 2 to transduce wingless signalling, Nature 400, 281-4 (1999).

18 Lin X, Buff EM, Perrimon N, Michelson AM, Heparan sulfate proteoglycans are essential for FGF receptor signaling during Drosophila development, Development 126, 3715-23 (1999).
19 Bellaiche Y, The I, Perrimon N, Tout-velu is a drosophila homologue of the putative tumor suppressor EXT-1 and is needed for Hh diffusion, Nature 394, 85-8 (1998).

20 The I, Bellaiche Y, Perrimon N, Hedgehog movement is regulated through Tout-velu-dependent synthesis of a heparan sulfate proteoglycan, Mol Cell 4, 633-9 (1999).

21 Tsuda M, Kamimura K, Nakato H, Archer M, Staatz W, Fox B, Humphrey M, Olson S, Futch T, Kaluza V, Siegfried E, Stam L, Selleck SB, The cell-surface proteoglycan Dally regulates Wingless signalling in Drosophila, Nature 400, 276-80 (1999).

22 Wodarz A, Nusse R, Mechanism of Wnt signaling in development, Annu Rev Cell Dev Biol 14, 59-88 (1998).

23 Siegfried E, Perrimon N, Drosophila Wingless: A paradigm for the function and mechanism of Wnt signaling, Bioessays 16, 395-404 (1994).

24 Sanson B, Generating patterns from fields of cells. Examples from drosophila segmentation, EMBO Rep 2, 1083-8 (2001).

25 Sanson B, Alexandre C, Fascetti N, Vincent JP, Engrailed and hedgehog make the range of Wingless assymetric in Drosophila embryos, Cell 98, 207-16 (1999).

26 Strigini M, Cohen SM, Wingless gradient formation in Drosophila wing, Curr Biol 10, 293-300 (2000).

27 Gerlitz O, Basler K, Wingful, an extracellular feedback inhibitor of Wingless, Genes Dev 16, 1055-9 (2002).

28 Pfeiffer S, Alexandre C, Calleja M, Vincent JP, Producing cells retain and recycle Wingless in Drosophila embryos, Curr Biol 10, 321-4 (2000).

29 Greco V, Hannus M, Eaton S, Argosomes: A potential vehicle for the spread of morphogens through epithelia, Cell 106, 633-45 (2001).

30 Ingham PW, McMahon AP, Hedgehog signaling in animal development: Paradigms and principles, Genes Dev 15, 3059-87 (2001).

31 Burke R, Nellen D, Bellotto M, Hafen E, Senti KA, Dickson BJ, Basler K, Dispatched, a novel sterol-sensing domain protein dedicated to the release of cholesterol modified hedgehog from signaling cells, Cell 99, 803-15 (1999).

32 Ingham PW, How cholesterol modulates the signal, Curr Biol 10, R180-3 (2000). 